\title{
Assessment of left atrial function in feline hypertrophic cardiomyopathy by using two- dimensional speckle tracking echocardiography
}

\author{
Arisara Kiatsilapanan and Sirilak Disatian Surachetpong ${ }^{*}$
}

\begin{abstract}
Background: Left atrial (LA) function plays an important role in diastolic dysfunction in cats with hypertrophic cardiomyopathy (HCM). Two-dimensional speckle tracking echocardiography (2D-STE) is a technique for assessing LA function. This study aimed to evaluate the LA function in HCM cats compared to normal cats, using 2D-STE.

Results: Seventeen client-owned cats affected with HCM and twenty healthy control cats were studied. Conventional echocardiographic and 2D-STE variables were measured and compared between groups (control and HCM groups). Variability of the peak atrial longitudinal strain (PALS) displayed good reproducibility with $4.7 \%$ intraobserver and 14\% inter-observer repeatability. The mean value of PALS in the HCM group (13.16 \pm 8.64) was lower than that in the control group $(28.54 \pm 10.31)(P<0.001)$. PALS was lowest in the LA roof region. The atrial longitudinal strains of septal and lateral regions were significantly lower in the HCM group than in the normal group. The PALS correlated with the percentage of the LA fractional shortening (LA-FS) $(r=0.538, P=0.001)$, the percentage of the LA ejection fraction (LA-EF) $(r=0.797, P<0.001)$, and the LA fractional area change (FAC) $(r=$ $0.746, P<0.001)$.
\end{abstract}

Conclusions: PALS is a feasible and reproducible method to evaluate the LA function in cats affected with HCM.

Keywords: Cats, Heart, Left atrium, Strain

\section{Background}

Hypertrophic cardiomyopathy ( $\mathrm{HCM})$ is one of the most common myocardial diseases. The prevalence of $\mathrm{HCM}$ in cats was approximately $10-15 \%$ in cats and increased with age [1-4]. The American College of Veterinary Internal Medicine consensus guideline of cardiomyopathies in cats suggests that left ventricular wall thickness during diastole of $\geq 6$ is indicative of left ventricular hypertrophy $[5,6]$. Hypertrophic cardiomyopathy is associated with diastolic dysfunction [5]. An advanced

\footnotetext{
* Correspondence: sirilakd27@gmail.com

Department of Veterinary Medicine, Faculty of Veterinary Science,

Chulalongkorn University, 10330 Bangkok, Thailand
}

diastolic dysfunction is related to clinical signs [2]. Some HCM cats may develop congestive heart failure, arterial thromboembolism, and sudden death [3, 7].

The left atrium (LA) plays an important role in cardiac performance through its three phasic functions: reservoir, conduit, and booster pump functions [8-12]. The first is the reservoir phase, in which the LA obtains blood from the pulmonary venous flow during the left ventricular systole. The second is the conduit phase, when the LA transfers blood passively into the left ventricle during early diastole. The final phase is the booster pump, which represents LA contraction during the late diastole [8-12]. Assessment of LA size is the most

(C) The Author(s). 2020 Open Access This article is licensed under a Creative Commons Attribution 4.0 International License, which permits use, sharing, adaptation, distribution and reproduction in any medium or format, as long as you give appropriate credit to the original author(s) and the source, provide a link to the Creative Commons licence, and indicate if changes were made. The images or other third party material in this article are included in the article's Creative Commons licence, unless indicated otherwise in a credit line to the material. If material is not included in the article's Creative Commons licence and your intended use is not permitted by statutory regulation or exceeds the permitted use, you will need to obtain permission directly from the copyright holder. To view a copy of this licence, visit http://creativecommons.org/licenses/by/4.0/ The Creative Commons Public Domain Dedication waiver (http://creativecommons.org/publicdomain/zero/1.0/) applies to the data made available in this article, unless otherwise stated in a credit line to the data. 
important indicater of the chronicity, severity and progression of the disease [5].

Several methods have been used in the assessment of LA function in humans and dogs, such as echocardiography, magnetic resonance imaging and computed tomography [13-19]. Echocardiographic techniques used for evaluating LA function in humans, dogs, and cats include phasic volume changes and tissue Doppler imaging, but these techniques have limitations, including load- and angledependence, and the tethering effect [5, 11, 14, 20-25].

Two-dimensional speckle tracking echocardiography (2D-STE) is an echocardiographic technique that can be used to assess LA function by tracking acoustic speckle patterns of the LA wall, and to analyse the myocardial motion $[10,11,26]$. Two-dimensional speckle tracking echocardiography is a feasible and reproducible method for evaluating LA wall deformation and can be used together with conventional echocardiography to assess LA function. [10, 11, 26].

Two-dimensional speckle tracking echocardiography has been utilized to assess LA function in humans and dogs [8-12, 26, 27]. Few studies using 2D-STE to assess left ventricular function in HCM cats have been published [22, 23, 28-30]. To our knowledge, there are no studies focusing on the assessment of LA function by 2D-STE in feline HCM. We hypothesized that changes in LA function of HCM cats can be detected using 2DSTE. This study aimed to use 2D-STE to evaluate changes in LA function in HCM cats and compare them to those of healthy control cats.

\section{Results}

A total of 37 cats were included in the study, including 20 control cats and $17 \mathrm{HCM}$ cats. The general characteristics of the control and HCM groups are summarized in Table 1. Age, body weight, heart rate and systolic blood pressure did not differ significantly between the control and HCM groups. Male and domestic shorthair cats were over-represented, but the number of cats in each sex and breed did not differ significantly between the control and HCM groups. Five of 17 cats in the HCM group had systolic anterior motion assessed by echocardiography. None of cats recruited to the study had mitral valve dysplasia which may be the cause of left ventricular outflow tract obstruction and left ventricular hypertrophy.

The result of the conventional echocardiography showed an increase in LAD and the ratio of LA to aorta dimension in the HCM group $(P<0.001)$ compared to the control group, while left ventricular internal dimension at end-diastole were significantly lower in the HCM group than in the control group $(P=0.027)$. The pulsedwave Doppler echocardiography demonstrated that peak
Table 1 The general characteristics of cats in the control and hypertrophic cardiomyopathy groups

\begin{tabular}{llll}
\hline Variable & $\begin{array}{l}\text { Control } \\
(\mathbf{N = 2 0 )}\end{array}$ & $\begin{array}{l}\text { HCM } \\
(\mathbf{N = 1 7 )}\end{array}$ & -value \\
\hline Age (year) & $5.05 \pm 3.03$ & $5 \pm 3.43$ & 0.963 \\
Body weight (Kg) & $4.47 \pm 0.92$ & $4.25 \pm 1.04$ & 0.497 \\
Sex & 14 & 10 & 0.512 \\
Male & 6 & 7 & \\
Female & & & 0.798 \\
Heart rate (bpm) & $206 \pm 20$ & $209 \pm 31$ & 0.073 \\
Systolic blood pressure (mmHg) & $128 \pm 20$ & $116 \pm 20$ & 0.843 \\
Breed & 10 & 10 & \\
Domestic Shorthair & 5 & - & \\
American shorthair & 1 & - & \\
Sphinx & 2 & - & \\
Scottish fold & 1 & - & \\
Siamese & 1 & 5 & \\
Exotic shorthair & - & 1 & \\
Persian & - & & \\
Khao Manee & & & \\
\hline Age body weight, & & & \\
\hline
\end{tabular}

Age, body weight, heart rate and systolic blood pressure are expressed as mean \pm standard deviation

Sex and breeds are expressed as the number of cats

Numerical data were compared by using the independent student t-test

Categorical data were compared by using Fisher's Exact test

HCM Hypertrophic cardiomyopathy

velocity of early diastolic transmitral flow $(P=0.002)$, peak velocity of systolic pulmonary vein flow $(P=0.001)$, and peak velocity of diastolic pulmonary vein flow $(P=$ 0.006 ) were significantly lower in the HCM group than the control group. The pulsed-wave Doppler echocardiography and tissue Doppler imaging were not significantly different between the two groups. The LA-FS $(P<0.001)$, LA-EF $(P=0.001)$ and FAC $(P<0.001)$ were significantly lower in the HCM group than in the control group (Table 2).

The PALS was significantly lower in the HCM group than the control group $(P<0.001)$. The longitudinal strain of LA regions was significantly reduced in the HCM group compared to the normal group, except the septal-roof and the lateral- roof of the LA (Table 3) (Fig. 1).

An assessment of the LA function of 3 subgroups [control group $(n=20)$, HCM cats with a LA diameter < $16 \mathrm{~mm}(n=09)$, and HCM cats with a LA diameter $\geq$ $16 \mathrm{~mm}(n=08)]$ was compared (Table 4$)$. The results showed that LA-FS $(P<0.001)$, LA-EF $(P=0.001)$, FAC $(P<0.001)$ and PALS $(P<0.001)$ in both HCM cat subgroups were significantly lower than in the control group. However, the values of these variables were not significantly different between HCM cat subgroups.

The correlations between the peak atrial longitudinal strain and echocardiographic values assessed by conventional echocardiography in entire population showed 
Table 2 Comparison of conventional echocardiographic values in the control and hypertrophic cardiomyopathy groups

\begin{tabular}{|c|c|c|c|}
\hline Variable & Control $(N=20)$ & HCM $(N=17)$ & $p$-value \\
\hline \multicolumn{4}{|c|}{ Size and structure } \\
\hline IVSd(mm) & $4.24 \pm 0.89$ & $7.12 \pm 1.14$ & $<0.001^{*}$ \\
\hline $\operatorname{LVIDd}(\mathrm{mm})$ & $14.31 \pm 1.65$ & $12.38 \pm 3.03$ & $0.027^{*}$ \\
\hline LVPWd(mm) & $3.5 \pm 0.59$ & $5.56 \pm 1.64$ & $<0.001^{*}$ \\
\hline IVSs(mm) & $7.18 \pm 1.40$ & $8.63 \pm 1.86$ & $0.01^{*}$ \\
\hline LVIDs(mm) & $6.35 \pm 1.63$ & $6.06 \pm 2.38$ & 0.669 \\
\hline LVPWs(mm) & $7.01 \pm 0.92$ & $8.24 \pm 1.94$ & $0.026^{*}$ \\
\hline $\mathrm{LA}(\mathrm{mm})$ & $12.22 \pm 1.42$ & $15.55 \pm 2.81$ & $<0.001^{*}$ \\
\hline Ao (mm) & $8.66 \pm 1.3$ & $8.3 \pm 1.73$ & 0.476 \\
\hline LA:Ao & $1.44 \pm 0.28$ & $1.92 \pm 0.44$ & $<0.001^{*}$ \\
\hline \multicolumn{4}{|l|}{ LV function } \\
\hline FS\% & $55.58 \pm 10.22$ & $51.38 \pm 10.79$ & 0.233 \\
\hline$E(\mathrm{~m} / \mathrm{s})$ & $0.83 \pm 0.19$ & $0.62 \pm 0.18$ & $0.002^{*}$ \\
\hline$A(m / s)$ & $0.61 \pm 0.12$ & $0.53 \pm 0.29$ & 0.346 \\
\hline $\mathrm{E}: \mathrm{A}$ & $1.34 \pm 0.31$ & $1.46 \pm 0.74$ & 0.09 \\
\hline IVRT (m/s) & $45.7 \pm 6.64$ & $47.4 \pm 9.12$ & 0.504 \\
\hline $\mathrm{S}(\mathrm{cm} / \mathrm{s})$ & $0.46 \pm 0.09$ & $0.31 \pm 0.13$ & $0.001^{*}$ \\
\hline $\mathrm{D}(\mathrm{cm} / \mathrm{s})$ & $0.35 \pm 0.06$ & $0.26 \pm 0.12$ & $0.006^{*}$ \\
\hline $\mathrm{AR}(\mathrm{cm} / \mathrm{s})$ & $0.14 \pm 0.03$ & $0.16 \pm 0.05$ & 0.367 \\
\hline S:D ratio & $1.25 \pm 0.2$ & $1.2 \pm 0.22$ & 0.464 \\
\hline$E^{\prime}(\mathrm{m} / \mathrm{s})$ & $0.11 \pm 0.03$ & $0.08 \pm 0.04$ & 0.056 \\
\hline$A^{\prime}(m / s)$ & $0.08 \pm 0.03$ & $0.07 \pm 0.04$ & 0.54 \\
\hline $\mathrm{S}^{\prime}(\mathrm{m} / \mathrm{s})$ & $0.07 \pm 0.02$ & $0.07 \pm 0.03$ & 0.625 \\
\hline$E^{\prime}: A^{\prime}$ ratio & $1.33 \pm 0.31$ & $1.15 \pm 0.39$ & 0.16 \\
\hline E:E' ratio & $7.8 \pm 2.92$ & $9.7 \pm 6.8$ & 0.265 \\
\hline \multicolumn{4}{|l|}{ LA function } \\
\hline LA-FS (\%) & $27.27 \pm 6.94$ & $14.95 \pm 8.23$ & $<0.001^{*}$ \\
\hline LA-EF (\%) & $69.10 \pm 13.64$ & $46.09 \pm 21.48$ & $0.001^{*}$ \\
\hline FAC (\%) & $63.73 \pm 11.30$ & $35.54 \pm 18.07$ & $<0.001^{*}$ \\
\hline
\end{tabular}

$A$ peak velocity of early diastolic transmitral flow, $A^{\prime}$ peak velocity of diastolic mitral annular motion as determined by pulsed wave Doppler, Ao Aorta, $A R$ peak velocity of pulmonary vein flow reversal at atrial contraction, $D$ peak velocity of diastolic pulmonary vein flow, $E$ peak velocity of early diastolic transmitral flow, $E^{\prime}$ peak velocity of early diastolic mitral annular motion as determined by pulsed wave Doppler, $E: A$ ratio of $E$ to $A, E^{\prime} A^{\prime}$ ratio of $E^{\prime}$ to $A^{\prime}$, $E: E^{\prime}$ ratio of $E$ to $E^{\prime}, F A C$ left atrial fractional area change, $F S$ left ventricular fractional shortening, HCM hypertrophic cardiomyopathy, IVRT isovolumic (or isovolumetric) relaxation time, IVSd interventricular septum thickness at enddiastole, IVSs interventricular septum thickness at end-systole, $L A$ left atrium, $L A: A o$ left atrial and aorta ratio, $L A-E F$ left atrial ejection fraction, $L A-F S$ left atrial fractional shortening, LVIDd left ventricular internal dimension at end -diastole, LVIDs left ventricular internal dimension at end -systole, LVPWd left ventricular posterior wall thickness at end-diastole, LVPWs left ventricular posterior wall thickness at end-systole, $S$ peak velocity of systolic pulmonary vein, $S^{\prime}$ peak velocity of systolic mitral annular motion as determined by pulsed wave Doppler, $S: D$ ratio of $S$ to $D$

Data are expressed as mean \pm standard deviation

* indicate statistical significance at $p<0.05$ assessed by the independent student t-test
Table 3 Two-dimensional echocardiographic data of cats in the control and hypertrophic cardiomyopathy groups

\begin{tabular}{llll}
\hline Variable & Control $(\boldsymbol{N}=\mathbf{2 0})$ & HCM $(\boldsymbol{N}=\mathbf{1 7})$ & $\boldsymbol{p}$-value \\
\hline PALS & $27.25[21.18,32.97]$ & $13.33[5.53,17.32]$ & $<0.001^{*}$ \\
Longitudinal strain of each left atrial region & & \\
Septal - base & $24.01[15.84,40.74]$ & $12.30[5.22,19.01]$ & $0.006^{*}$ \\
Septal - mid & $32.12[25.67,54.54]$ & $17.07[6.48,24.82]$ & $<0.001^{*}$ \\
Septal - roof & $17.18[6.22,29.59]$ & $7.57[3.23,13.13]$ & 0.063 \\
Lateral - roof & $18.43[6.86,32.19]$ & $4.00[-0.22,28.47]$ & 0.067 \\
Lateral- mid & $23.30[18.17,35.77]$ & $15.83[1.97,25.42]$ & $0.038^{*}$ \\
Lateral- base & $37.42[13.60,46.80]$ & $10.8[7.22,17.23]$ & $0.002^{*}$ \\
\hline
\end{tabular}

PALS peak atrial longitudinal strain, HCM hypertrophic cardiomyopathy

Data are expressed as median and 25th, 75th percentiles

* indicate statistical significance at $p<0.05$ by Mann-Whitney U Test

weak positive correlations between PALS and peak velocity of early diastolic transmitral flow $(\mathrm{r}=0.41, P=$ $0.012)$, peak velocity of systolic pulmonary vein flow $(\mathrm{r}=$ $0.41, P=0.013)$, peak velocity of diastolic pulmonary vein flow $(\mathrm{r}=0.341, P=0.042)$, and peak velocity of early diastolic mitral annular motion as determined by pulsed-wave Doppler echocardiography $(r=0.459, P=$ 0.004). Moderate negative correlations between PALS and interventricular septum thickness at end-diastole $(\mathrm{r}=-0.563, P<0.001)$ and left ventricular posterior wall thickness at end-diastole $(\mathrm{r}=-0.516, P=0.001)$ were observed. There was no significant correlation between PALS and LAD (Table 5). There was also no correlation between PALS and heart rate $(\mathrm{r}=-0.042, P=0.803)$.

The correlation analysis of data of all cats demonstrated a highly positive correlation between PALS and LA-EF $(\mathrm{r}=0.797, P<0.001)$ as well as PALS and FAC $(\mathrm{r}=0.746$, $P<0.001)$. The peak atrial longitudinal strain moderately correlated with LA-FS $(0.538, P=0.001)$ (Fig. 2).

The ANCOVA test demonstrated that age, sex and breeds had no effect on values of PALS, LA-FS and LAEF. However, FAC was affected by breeds (domestic shorthair or pure breeds) $(P=0.04)$.

The intra-observer and inter-observer measurements of PALS variability were $4.17 \%$ and $14 \%$, respectively.

\section{Discussion}

We undertook this study to assess LA deformation of control cats and cats affected with HCM, using 2DSTE. The first major finding of this study was that 2D-STE is feasible for use in assessing LA deformation in cats. The second was that a difference in PALS was identified between cats with HCM and healthy cats. Finally, no correlation was found between PALS and LA size, but it correlated well with other echocardiographic parameters of LA function variables, including LA-FS, LA-EF and FAC. 


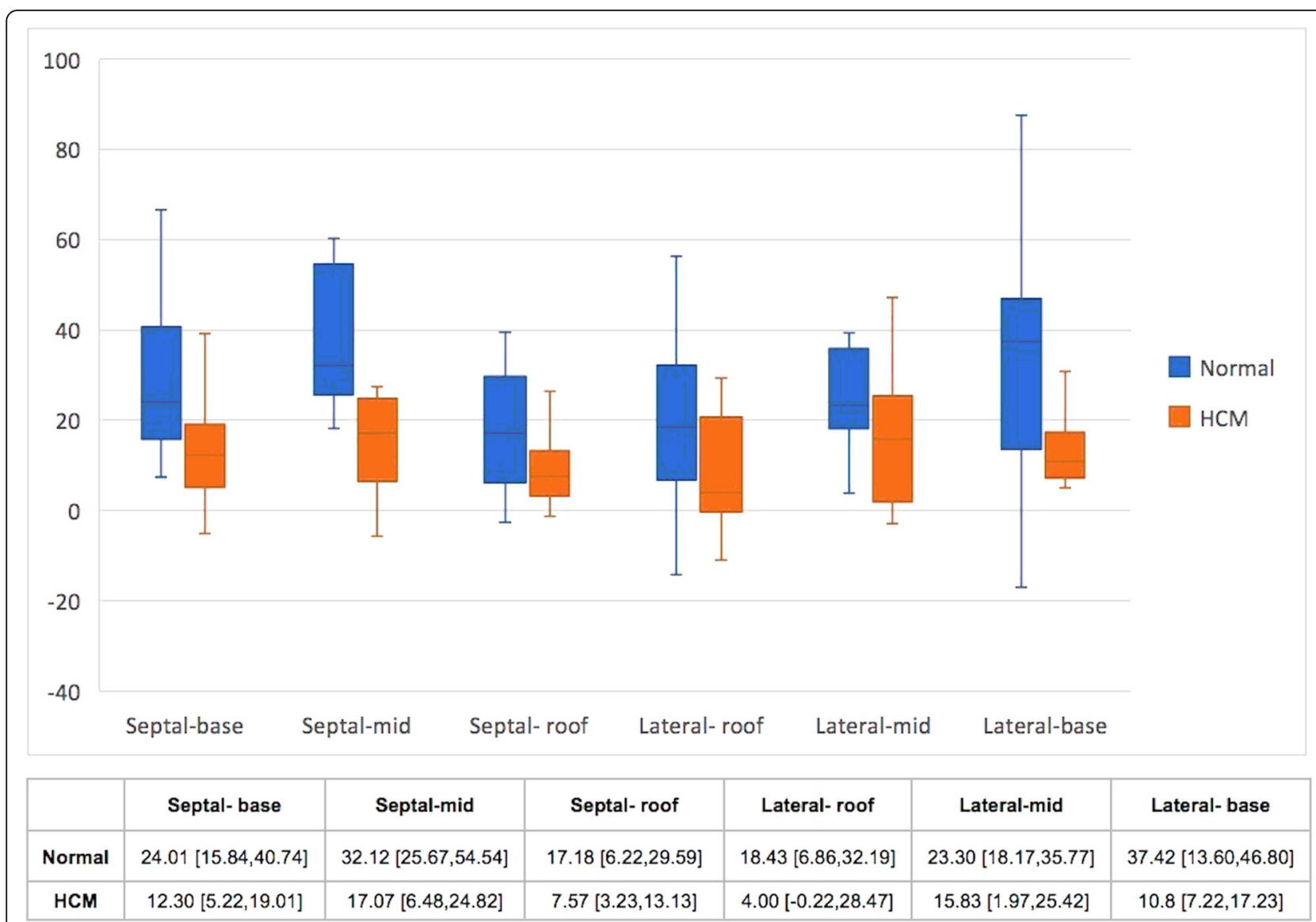

Fig. 1 The Box and Whisker plot shows median and 25 to 75 percentile range of the atrial longitudinal strain in each region between the normal (blue) and hypertrophic cardiomyopathy (HCM) groups (orange)

Two-dimensional speckle tracking echocardiography is an echocardiographic technique that can be used to assess LA longitudinal strain in dogs $[8,9,12,31]$ and humans $[10,11,27,32]$. Few studies using 2D-STE to assess left ventricular function in cats with HCM have been published [22, 23, 28-30]. This technique has been used to evaluate left ventricular myocardial function with adequate repeatability $[22,23,28]$. To our knowledge, no study focusing on assessment of LA function in feline HCM using 2D-STE has been reported. The present study demonstrated that 2D-STE may be used to evaluate changes in LA function in HCM cats with high repeatability and reproducibility. Intra- and inter-observer variability of PALS were clinically acceptable $(\mathrm{CV}<15 \%)$, and similar results have been reported in humans [27] and dogs [8, 12].

Table 4 Assessment of left atrial function of 3 subgroups of cats

\begin{tabular}{|c|c|c|c|c|}
\hline Variables & $\begin{array}{l}\text { Control } \\
(N=20)\end{array}$ & $\begin{array}{l}\text { HCM with } \\
\text { LAD }<16 \mathrm{~mm} \\
(N=09)\end{array}$ & $\begin{array}{l}\text { HCM with } \\
\text { LAD } \geq 16 \mathrm{~mm} \\
(N=08)\end{array}$ & $P$-value \\
\hline LA-FS (\%) & $25.27[23.29,31.67]^{a}$ & $17.4[8.68,19.67]^{b}$ & $14.26[6.38,20.00]^{b}$ & $<0.001^{*}$ \\
\hline LA-EF (\%) & $64.97[59.75,83.05]^{a}$ & $36.53[28.07,75.9]^{b}$ & $39.87[25.45,60.41]^{b}$ & $0.002^{*}$ \\
\hline FAC (\%) & $63.28[57.46,72.31]^{\mathrm{a}}$ & $29.57[14.66,51.50]^{b}$ & $32.09[24.07,52.39]^{b}$ & $<0.001^{*}$ \\
\hline PALS (\%) & $27.25[21.18,32.97]^{a}$ & $8.4[5.53,21.57]^{b}$ & $14.50[5.94,18.34]^{b}$ & $<0.001^{*}$ \\
\hline
\end{tabular}

FAC left atrial fractional area change, HCM hypertrophic cardiomyopathy, LAD left atrial diameter, LA-EF left atrial ejection fraction, LA-FS left atrial fractional shortening, PALS peak atrial longitudinal strain

Data are expressed as mean \pm standard deviation

*indicate statistical significance at $P<0.05$ of 3 subgroups

The significance difference was assessed by Kruskal Wallis test

${ }^{\mathrm{a}}$ and ${ }^{\mathrm{b}}$ indicate significant difference 
Table 5 The correlation of the peak atrial longitudinal strain and echocardiographic values assessed by conventional echocardiography in entire population

\begin{tabular}{|c|c|c|}
\hline Variable & $r$ & $p$-value \\
\hline \multicolumn{3}{|c|}{ Size and structure } \\
\hline IVSd (mm) & -0.563 & $<0.001^{*}$ \\
\hline LVIDd (mm) & 0.362 & $0.028^{*}$ \\
\hline LVPWd (mm) & -0.516 & $0.001^{*}$ \\
\hline $\mathrm{LA}(\mathrm{mm})$ & -0.248 & 0.139 \\
\hline LA:Ao & -0.315 & 0.058 \\
\hline \multicolumn{3}{|l|}{ LV function } \\
\hline FS\% & -0.061 & 0.721 \\
\hline$E(m / s)$ & 0.41 & $0.012^{*}$ \\
\hline$A(m / s)$ & 0.277 & 0.107 \\
\hline$E: A$ & -0.191 & 0.271 \\
\hline IVRT (m/s) & -0.204 & 0.240 \\
\hline $\mathrm{S}(\mathrm{cm} / \mathrm{s})$ & 0.41 & $0.013^{*}$ \\
\hline $\mathrm{D}(\mathrm{cm} / \mathrm{s})$ & 0.341 & $0.042^{*}$ \\
\hline $\mathrm{AR}(\mathrm{cm} / \mathrm{s})$ & 0.164 & 0.353 \\
\hline S:D ratio & 0.164 & 0.338 \\
\hline $\mathrm{E}^{\prime}(\mathrm{cm} / \mathrm{s})$ & 0.459 & $0.004^{*}$ \\
\hline$A^{\prime}(\mathrm{cm} / \mathrm{s})$ & 0.117 & 0.523 \\
\hline $\mathrm{S}^{\prime}(\mathrm{cm} / \mathrm{s})$ & 0.078 & 0.651 \\
\hline$E^{\prime}: A^{\prime}$ ratio & 0.132 & 0.435 \\
\hline E:E' ratio & -0.21 & 0.212 \\
\hline
\end{tabular}

Abbreviations: $A$ peak velocity of early diastolic transmitral flow, $A^{\prime}$ peak velocity of diastolic mitral annular motion as determined by pulsed wave Doppler, $A R$ Peak velocity of pulmonary vein flow reversal at atrial contraction, $D$ Peak velocity of diastolic pulmonary vein flow, $E$ Peak velocity of early diastolic transmitral flow, $E^{\prime}$ Peak velocity of early diastolic mitral annular motion as determined by pulsed wave Doppler, $E: A$ Ratio of $E$ to $A, E^{\prime} A$ ' Ratio of $E^{\prime}$ to $A^{\prime}, E$ :E' Ratio of $E$ to $E$ ', FAC Left atrial fractional area change, FS Left ventricular fractional shortening, HCM Hypertrophic cardiomyopathy, IVRT Isovolumic (or isovolumetric) relaxation time, IVSd Interventricular septum thickness at end-diastole, LA Left atrium, LA:Ao Left atrial and aorta ratio, LV Left ventricle, LVIDd Left ventricular internal dimension at end -diastole, LVPWd Left ventricular posterior wall thickness at end-diastole, $S$ Peak velocity of systolic pulmonary vein, $S^{\prime}$ Peak velocity of systolic mitral annular motion as determined by pulsed wave Doppler, $S: D$ Ratio of $S$ to $D$

The significant correlation was assessed by Pearson's correlation coefficient *indicate statistical significance at $p<0.05$

The PALS, assessed by 2D-STE provides information on the longitudinal deformation of the LA during the reservoir phase $[10,11]$. The result of this study showed that PALS was lower in the HCM group than the control group. In addition, LA-FS, LA-EF and FAC, conventional echocardiographic parameters for assessing LA contraction, were found to be lower in the HCM group than in the control group. This result is in agreement with previous studies reporting a reduction in LA function in cats affected with HCM, particularly those with congestive heart failure [5, 20,33]. The present study showed that the LA roof had the lowest longitudinal strain. The LA roof is closed to the mediastinum, which may limit the movement of this region [26]. In humans, the strains of base and mid regions are similar [34]. Our study showed that the mid region had the highest strain. Further investigations are needed to clarify whether species variation is the cause of this difference. The longitudinal strains of all LA regions were decreased in the HCM group, suggesting global LA reservoir functional changes in cats affected with HCM. In this study, only one peak of atrial longitudinal strain was found in cats, which is different from that found in humans and dogs [8-11,31]. This may occur secondary to rapid heart rate in cats. As in case of pulsed-wave Doppler echocardiography and tissue Doppler imaging, early and late diastolic transmitral flow and early and late diastolic mitral annular motion can be summated. Two cats from the control and HCM groups had early and late diastolic transmitral flow and mitral annular motion summation.

Interestingly, the present study showed that changes in PALS, LA-FS, LA-EF and FAC were found in HCM cats with both normal and enlarged LA size. A previous study showed a decrease in LA-EF in HCM cats with congestive heart failure with significant LA enlargement but not in asymptomatic HCM cats [5]. Whether a decrease in LA function is a consequence or cause of LA dilatation has to be proved. The results of our study suggest that the poor performance of the LA function in HCM cats may be not dependent on the LA size because the LA function and deformation have been detected in HCM cats both with and without LA enlargement.

The PALS assessed by 2D-STE correlated with LA function parameters assessed by conventional echocardiography. This result suggests that PALS offers an additional method for evaluating LA function. However, the loaddependency and high repeatability are limitations that must be addressed for the routine use 2D-STE [10, 11, 32, 34]. Based on the ANCOVA of data from this study, PALS was not affected by age, breed or sex. However, breed did affect FAC when assessed by conventional echocardiography. These findings suggest that PALS may be a more suitable technique for evaluating LA function in cats than some parameters assessed by conventional echocardiography. However, this study could not demonstrate the superiority of PALS as an early detection method because the decline in PALS was detected at the same time as the decreases in LA-FS, LA-EF and FAC. The usefulness of 2D-STE as an early detection method for the assessment of cardiac dysfunction needs further studies.

PALS also correlated with left ventricular wall thickness, chamber size and diastolic function parameters assessed by conventional echocardiography and tissue Doppler imaging. This finding suggests a relationship between left ventricular structural and functional changes and LA reservoir function. 


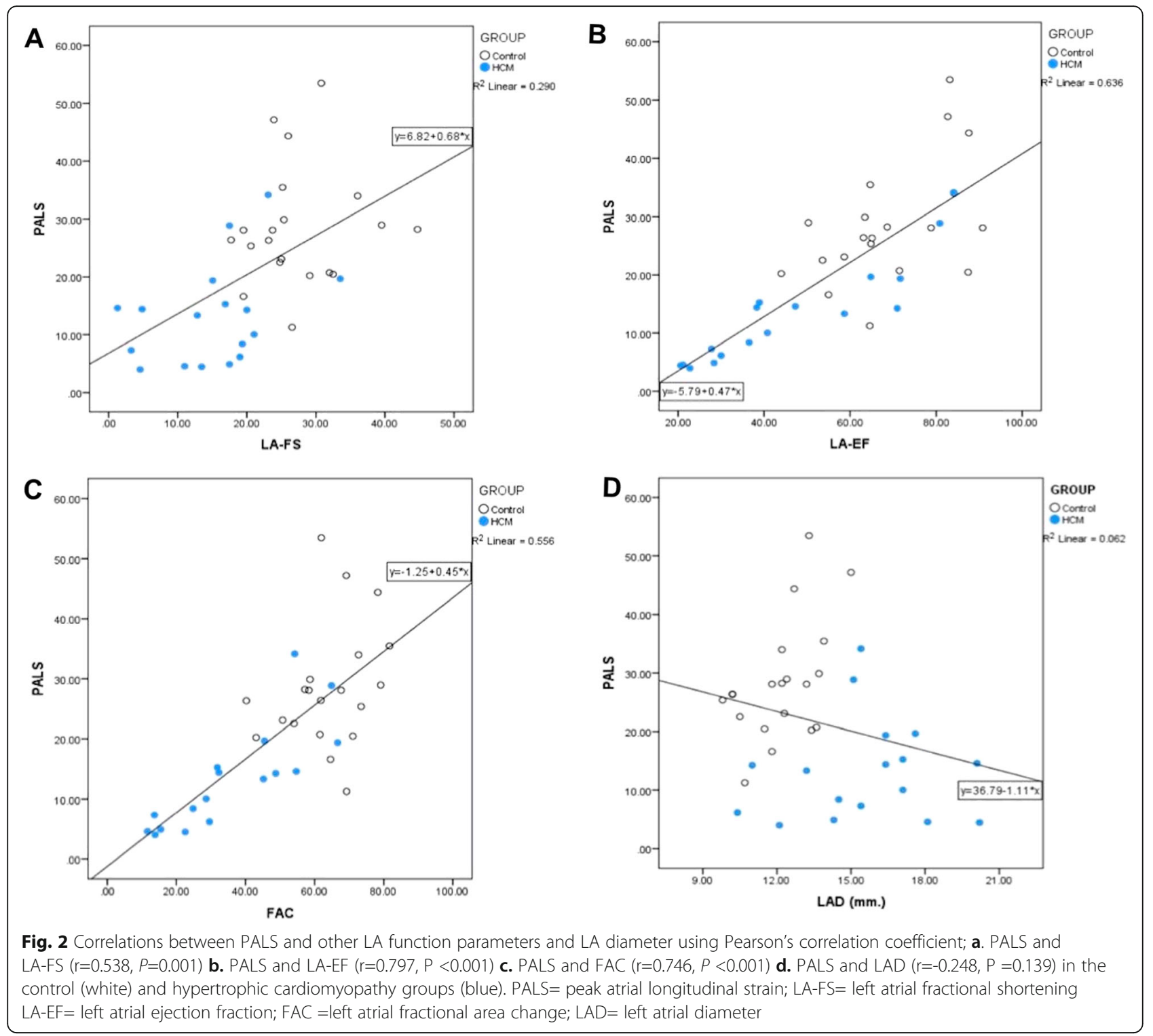

The present study has some limitations that should be considered. First, the number of cats used was relatively small. Studies with a larger number of cats should be performed before adapting 2D-STE for use in clinical routine. Second, 2D-STE software was created for the analysis of left ventricular function in humans; therefore, it may have some limitations for LA function assessment in cats. In addition, the inter-vendor software variability is high; therefore, the results of this study can only be compared by using the same STE software. Third, during assessment it was challenging to trace the thin feline LA wall and therefore also some undesired tracking of extracardiac structure (such as mediastinum) could have occurred; however, increasing the size by increasing the depth and gain of images may help to visualize the LA wall. Fourth, myocardial wall dropout in the interatrial septum and pulmonary vein inlets may affect the tracking procedure. Tracing the LA cavity before atrial contraction may help to visualize these areas better [9]. Lastly, 2D-STE requires high-quality images that are difficult to obtain in cats with a very fast heart rate. The 2D-STE software used in the present study does not allow analysis in subjects with heart rate higher than 240 beats/minute. Therefore, cats had to be handled in low-stress conditions to help maintain a low heart rate. The use of an ultrasound machine system with higher frame rate may solve this problem [35]. Although a rapid heart rate may affect the image and tracking quality, the results of this study showed that PALS did not correlate with heart rate, suggesting that heart rate may not have a direct effect on PALS. 


\section{Conclusions}

Assessment of the PALS of the LA by 2D-STE is feasible to evaluate LA deformation in HCM cats. The method showed an acceptable repeatability and reproducibility. Studies with a larger number of HCM cats should be performed to confirm the advantages of 2D-STE in the assessment of LA function before the technique can be applied in the routine clinical settings.

\section{Methods}

\section{Animals}

The study population consisted of healthy cats and client-owned cats affected with HCM. All cats presented at Small Animal Teaching Hospital, Faculty of Veterinary Science, Chulalongkorn University, Thailand during August 2018 - June 2019. The study protocol was approved by the Animal Care and Use Committee, Faculty of Veterinary Science, Chulalongkorn University, Thailand (Animal Use Protocol No.1,831,073). The sample size for control and HCM groups (at least 17 cats per group) was calculated by the statistical software ( $G$ * Power test 3.1), using the estimated standard deviation of the percentage of the LA fractional shortening (LA-FS) from a previous study [5], an expected $80 \%$ power, and $\alpha$ value of 0.05 , which was sufficient to determine a minimum difference in LA-FS between the two groups. LA-FS was chosen because of a lower standard deviation compared to other echocardiographic parameters.

\section{Cats}

Adult cats ( $>1$ year old) with body weight 2-6 kilograms, any sex and breed were enrolled in the study. Clinical findings for all cats were recorded. All cats were subject to a complete physical examination, systolic blood pressure measurement and blood collection for complete blood count, blood chemistry and total T4 measurements. Cardiac examination including echocardiography, electrocardiography and thoracic radiography was performed without sedation in all cats. No cats had received medications before being enrolled in the study. Cats with renal disease (creatinine $>2.0 \mathrm{mg} / \mathrm{dL}$ ), systemic hypertension (systolic blood pressure $>160 \mathrm{mmHg}$ ), hyperthyroidism (serum total T4 concentration $>4 \mu \mathrm{g} / \mathrm{dl}$ ) [36] and any systemic diseases were excluded from the study.

Cats were divided into two groups: the control and HCM groups. Those in the control group consisted of healthy cats that had a left ventricular wall thickness of $<6 \mathrm{~mm}$ and an LA diameter of $<16 \mathrm{~mm}$ assessed by echocardiography and showed no other cardiovascular and systemic illness. Cats with left ventricular wall thickness of $\geq 6 \mathrm{~mm}$ during end diastole were recruited into the HCM group [4-7, 30, 36, 37]. Cats with and without congestive heart failure signs were accepted. For subgroups analysis, the population consisted of three subgroups (control group, HCM cats with a LA diameter $<16 \mathrm{~mm}$, and HCM cats with a LA diameter $\geq$ $16 \mathrm{~mm})$.

\section{Conventional echocardiography}

Two-dimensional and M-mode echocardiography were performed by an investigator (SS). An ultrasound machine (Eko7, Samsung Medison, Seoul, South Korea) with a 4-12 MHz phased array transducer was used. Mmode echocardiography was performed on the right parasternal long-axis four-chamber view, to measure the chamber size and wall thickness by using a leading edgeto-leading edge technique. The M-mode cursor placed perpendicular to the interventricular septum and left ventricular wall below the tips of the mitral valves at the largest ventricular chamber size. Left ventricular internal dimension at end-diastole and end-systole, interventricular septum thickness at end-diastole and end-systole, left ventricular posterior wall thickness at end-diastole and end-systole were recorded. The percentage of left ventricular fractional shortening (FS\%) was calculated by subtracting the left ventricular systolic dimension from the diastolic dimension dividing by the diastolic dimension. The ratio of LA to aorta dimension was measured during first diastolic frame of aortic valve closure from a right parasternal short axis view using two-dimensional echocardiography [20].

Pulsed-wave Doppler and tissue Doppler imaging were used for assessing left ventricular diastolic function [6, 22]. Transmitral flow velocities were measured from the left apical four-chamber view. The gate was placed at the tips of the mitral valve leaflets when they were wide open [38]. Peak velocity of early diastolic transmitral flow, peak velocity of late transmitral flow and the ratio of peak velocity of early diastolic to late diastolic transmitral flow was recorded. Isovolumic (or isovolumetric) relaxation time was measured from the left apical five-chamber view by placing the gate in the left ventricular outflow tract near the anterior mitral valve leaflet to reveal both aortic ejection flow and left ventricular inflow [37, 39]. Pulmonary vein flow velocities were measured in the right parasternal short-axis view $[39,40]$. Peak velocity of systolic and diastolic pulmonary vein flow, and flow reversal at atrial contraction were recorded. The ratio of peak velocity of systolic to diastolic pulmonary vein flow were calculated. The myocardial motion along the longitudinal axis of the heart was investigated by placing the gate on the subendocardial portions of the lateral corner of the mitral annulus [24]. Peak velocity of early and late diastolic mitral annular motion and the ratio of peak velocity of early to late diastolic mitral annular motion as determined by pulsed-wave Doppler echocardiography were recorded. The ratio of peak velocity of early diastolic 


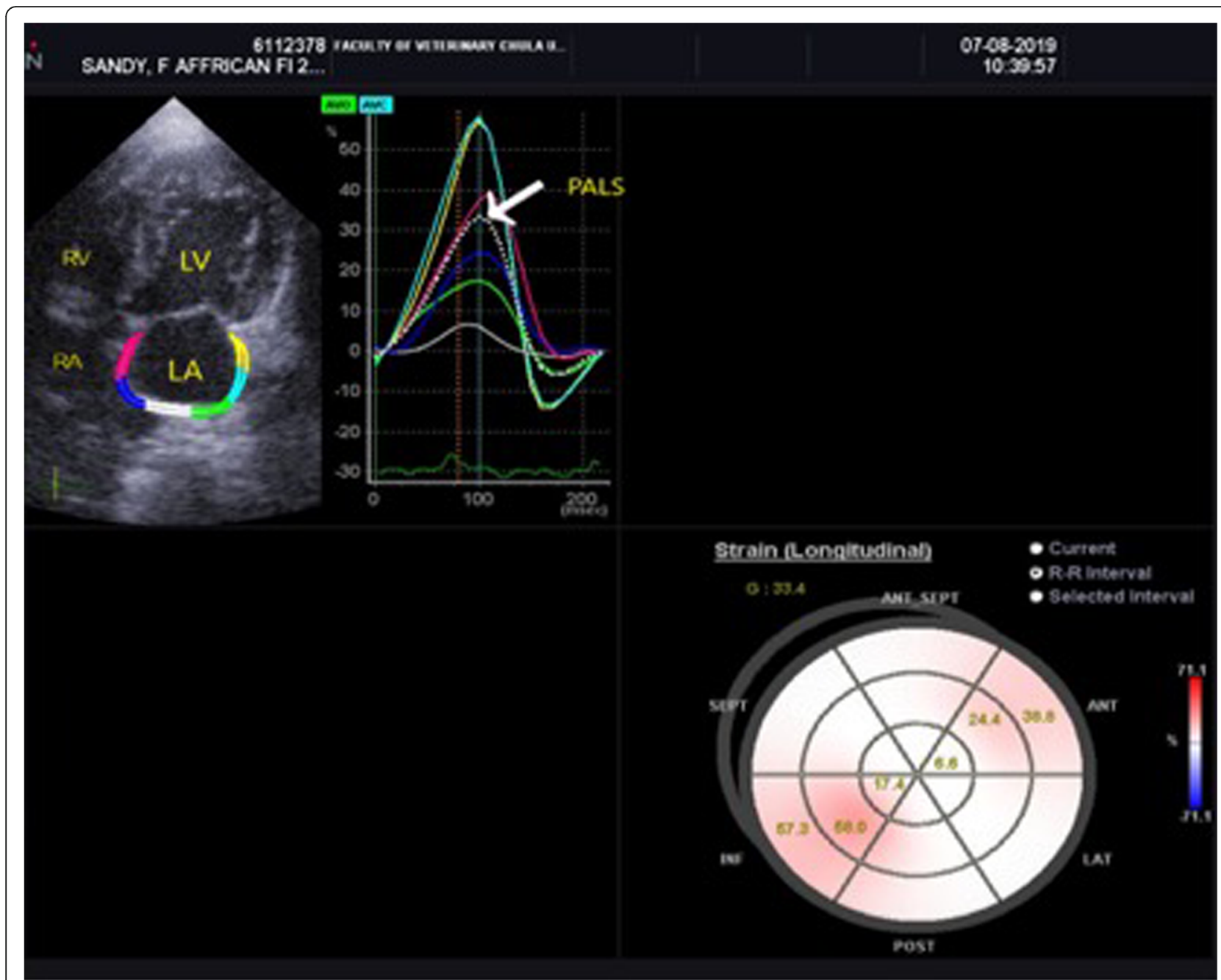

Fig. 3 The image of the left apical four-chamber view of the left atrial strain profile of a hypertrophic cardiomyopathy cat. A region of interest is manually drawn to include the left atrial wall. The automatic software system divided the left atrial wall into 6 different segments with different colors. A white dotted line is presented as the mean of strain value of the left atrium. $L A=l e f t$ atrium; $L V=l e f t$ ventricle; PALS= peak atrial longitudinal strain (white arrow); $\mathrm{RA}=$ right atrium; $\mathrm{RV}=$ right ventricle

transmitral flow to peak velocity of early diastolic mitral annular motion were calculated [25]. The summation of early and late diastolic transmitral flows and early and late diastolic mitral annular motion assessed by pulsed-wave Doppler echocardiography was excluded from the analysis.

The LA diameter (LAD) was measured on the right parasternal long-axis four-chamber view parallel with the mitral annulus [40]. The maximal and minimal LAD (LADmax and LADmin) were measured. The LADmax was measured at end-systole (one frame before opening of mitral valve), and the LADmin was measured at peak atrial contraction (one frame before closure of mitral valve) [5]. Changes of the LAD were expressed as the percentage of fractional shortening of the left atrium (LA-FS) by the formula (LADmaxLADmin/LADmax) x100. The left apical four-chamber view was used to measure the maximal LA volume during atrial end-diastole and minimal LA volume during atrial endsystole. The percentage of LA ejection fraction (LA-EF) was calculated by an ultrasound machine automated software [5]. The LA area change (FAC) was measured by tracing the LA endocardial border during LA end diastolic and systolic phases on left apical four- chamber view. Left atrial maximal area (LAAmax) and minimal area (LAAmin) were measured in $\mathrm{cm}^{2}$. Then, FAC was then calculated with the formula FAC $=[($ LAAmax - LAAmin $) /$ LAAmax $] \times 100[8,10]$. The measurements of LA-EF and FAC were performed on the same cardiac cycle as 2D-STE.

Two-dimensional speckle tracking echocardiography (2DSTE)

Two-dimensional speckle tracking echocardiography of the left apical four-chamber view was used to analyze the longitudinal deformation of the left atrium. Two- 
dimensional echocardiographic images were recorded at 100 frames/s for consecutive three cardiac cycles and three cineloops and stored these in the Digital Imaging and Communications in Medicine format. Offline analysis was performed in images with good quality from each cat. The LA wall, including the interatrial septum, the lateral wall and the atrial roof were tracked along during end-diastole. After automatic tracking, manual editing was performed to correct software errors in the region of interest. The ultrasound machine computer software (Strain 2.0 with Bull's Eye) calculated the LA strain. The mean values of the measurements from three consecutive cardiac cycles were used in all analyses. Six segments were analyzed in each cat. The strain of each segment (as percentages) was plotted on the $y$-axis versus time (in seconds) on the $x$-axis over an entire cardiac cycle (Fig. 3). The different colored graphs represent strain curves from different segments. The white dotted line is the global strain. The QRS complex was used as the zero reference point, the peak positive longitudinal strain corresponds to the atrial reservoir function, the strain during early diastole represents atrial conduit function, and strain during late diastole corresponds to atrial contractile function. The peak atrial longitudinal strain (PALS) was the average of the peak of strain curves at the end of reservoir phase $[8,10]$. The atrial strain rate was not analyzed in the present study.

\section{Measurement variability}

The data from six randomly selected cats in the control group were used for calculating the variability of PALS. For the intra-observer variability, the measurement data from the same operator repeated on two different days (seven days apart) were used. Measurements were performed in the same cardiac cycle from the same cine loop. The inter-observer variability was calculated from measurements of two operators with different levels of experience in echocardiography (SS: Diplomate of the Asian College of Internal Medicine (Cardiology) and $\mathrm{PhD}, \mathrm{AK}: \mathrm{MS})$. The variability was quantified as the coefficient of variation $(\mathrm{CV})$ by the formula, $\% \mathrm{CV}=$ standard deviation/mean $\mathrm{x} 100$. The degree of repeatability was determined as follows: $\mathrm{CV}<5 \%$, very low variability; $5-15 \%$, low variability; $16-25 \%$ moderate variability; or $>25 \%$ high variability $[8,41]$.

\section{Statistical analysis}

Statistical analyses were performed using a commercially available software (SPSS version 22, Inc, Chicago, IL, USA). Descriptive statistics were used to describe the characteristics of the cats including sex, breed, age, body weight, systolic blood pressure and heart rate. The normality of data was assessed with the Shapiro-Wilks normality test. Comparisons between the two groups (the control and HCM cats) were performed by using the independent student t-test for normally distributed data and the Mann-Whitney $U$ test for non-normally distributed data. The categorical data were compared by using Fisher's exact test. Comparisons of 3 subgroups, including control and $\mathrm{HCM}$ cats with $\mathrm{LAD}<16 \mathrm{~mm}$, and the $\mathrm{HCM}$ cats with LAD $>16 \mathrm{~mm}$, were performed by using the Kruskal-Wallis Test. The multiple comparisons were performed by the Duncan test method. An Analysis of Covariance model was used to test the fixed effects of sex, breed, and age, as covariates on conventional and 2D-STE-derived echocardiographic variables. The correlations between PALS and LA-FS, LA-EF, and FAC, assessed by conventional echocardiography, were tested by the Pearson's correlation coefficient. A value of $P<$ 0.05 was considered significant.

\section{Abbreviations \\ 2D-STE: Two-dimensional speckletracking echocardiography; CV: Coefficient of variation; FAC: Fractional area change; HCM: Hypertrophic cardiomyopathy; LA: Left atrium (atrial); LA-EF: The percentage of the LAejection fraction; LA-FS: The percentage of the LAfractional shortening; LAA: Left atrial area; LAD: Left atrial diameter; PALS: Peak atrial longitudinalstrain}

\section{Acknowledgements}

The authors would like to thank the Small Animal Hospital, Faculty of Veterinary Science, Chulalongkorn University for supporting data and facilities and Dr. Vachira Hunprasit for the statistical assistance.

\section{Authors' contributions}

Study conception and design: SDS; Acquisition of data: AK and SDS; Analysis of and interpretation of data: AK and SDS; Drafting of manuscript: AK and SDS; Critical revision: SDS; All authors read and approved the final manuscript.

\section{Funding}

This study was supported by the 90th Anniversary of Chulalongkorn University, Rachadapisek Sompote Fund and the 72nd anniversary of his Majesty King Bhumibala Aduladeja Fund, Graduate School, Chulalongkorn University, Thailand.

\section{Availability of data and materials}

The datasets used and/or analyzed during the current study are available from the corresponding author on reasonable request.

Ethics approval and consent to participate

The study protocol was approved by the Animal Care and Use Committee, Faculty of Veterinary Science, Chulalongkorn University, Thailand (Animal Use Protocol No.1,831,073). The owner gave written informed consent for their pets to be part of this study.

Consent for publication

Written informed consent was obtained from the cats' owners for publication of this manuscript.

\section{Competing interests}

The authors do not have any conflicts of interest to disclose.

Received: 9 March 2020 Accepted: 4 September 2020

Published online: 18 September 2020

\section{References}

1. Paige CF, Abbott JA, Elvinger F, Pyle RL. Prevalence of cardiomyopathy in apparently healthy cats. J Am Vet Med Assoc. 2009;234(11):1398-403. 
2. Payne J, Borgeat K, Connolly D, Boswood A, Dennis S, Wagner T, et al. Prognostic indicators in cats with hypertrophic cardiomyopathy. J Vet Intern Med. 2013;27(6):1427-36.

3. Fox PR, Keene BW, Lamb K, Schober KA, Chetboul V, Luis Fuentes V, et al. International collaborative study to assess cardiovascular risk and evaluate long-term health in cats with preclinical hypertrophic cardiomyopathy and apparently healthy cats: the REVEAL study. J Vet Intern Med. 2018;32(3):930-43.

4. Freeman LM, Rush JE, Stern JA, Huggins GS, Maron MS. Feline hypertrophic cardiomyopathy: a spontaneous large animal model of human HCM. Cardiovasc Res. 2017:8(4):139.

5. Linney C, Dukes-McEwan J, Stephenson H, López-Alvarez J, Fonfara S. Left atrial size, atrial function and left ventricular diastolic function in cats with hypertrophic cardiomyopathy. J Small Anim Pract. 2014;55(4):198-206.

6. Luis Fuentes V, Abbott J, Chetboul V, Côté E, Fox PR, Häggström J, et al. ACVIM consensus statement quidelines for the classification, diagnosis, and management of cardiomyopathies in cats. J Vet Intern Med. 2020;34(3):1062-77.

7. Silva A, Muzzi R, Oberlender G, Nogueira R, Muzzi L. Feline hypertrophic cardiomyopathy: an echocardiographic approach. Arch Med Vet. 2013;45(1):1-6.

8. Baron Toaldo M, Romito G, Guglielmini C, Diana A, Pelle N, Contiero B, et al. Assessment of left atrial deformation and function by 2 -dimensional speckle tracking echocardiography in healthy dogs and dogs with myxomatous mitral valve disease. J Vet Intern Med. 2017;31(3):641-9.

9. Caivano D, Rishniw M, Birettoni F, Patata V, Giorgi M, Porciello F. Left atrial deformation and phasic function determined by two-dimensional speckletracking echocardiography in dogs with myxomatous mitral valve disease. J Vet Cardiol. 2018;20(2):102-14.

10. Cameli M, Lisi M, Righini FM, Mondillo S. Novel echocardiographic techniques to assess left atrial size, anatomy and function. J Cardiovasc Ultrasound. 2012;10(1):4.

11. Cianciulli TF, Saccheri MC, Lax JA, Bermann AM, Ferreiro DE. Twodimensional speckle tracking echocardiography for the assessment of atrial function. World J Cardiol. 2010;2(7):163.

12. Dermlim A, Nakamura K, Morita T, Osuga T, Nisa K, Sasaoka K, et al. The repeatability and left atrial strain analysis obtained via speckle tracking echocardiography in healthy dogs. J Vet Cardiol. 2019;23:69-80.

13. Vizzardi E, D'aloia A, Rocco E, Lupi L, Rovetta R, Quinzani F, et al. How should we measure left atrium size and function? I Clin Ultrasound. 2012; 40(3):155-66.

14. Coelho M, Muzzi R, Abreu C, Schulien T, Muzzi L, Oliveira L, et al. Assessment of left atrial function in dogs with myxomatous mitral valve disease by biplane simpson's method. Arq Bras Med Vet Zoo. 2018;70(5): 1349-54.

15. Hoit BD. Assessment of left atrial function by echocardiography: novel insights. Curr Cardiol Rep. 2018;20(10):96.

16. Blume GG, Mcleod CJ, Barnes ME, Seward JB, Pellikka PA, Bastiansen PM, et al. Left atrial function: physiology, assessment, and clinical implications. Eur J Echocardiogr. 2011;12(6):421-30

17. Nakamura K, Osuga T, Morishita K, Suzuki S, Morita T, Yokoyama N, et al. Prognostic value of left atrial function in dogs with chronic mitral valvular heart disease. J Vet Intern Med. 2014;28(6):1746-52.

18. Bouvard J, Thierry F, Culshaw GJ, Schwarz T, Handel I, Pereira YM. Assessment of left atrial volume in dogs: comparisons of two-dimensional and real-time three-dimensional echocardiography with ECG-gated multidetector computed tomography angiography. J Vet Cardiol. 2019;24: 64-77.

19. Gan GC, Ferkh A, Boyd A, Thomas L. Left atrial function: evaluation by strain analysis. Cardiovasc Diagn Ther. 2018:8(1):29.

20. Abbott JA, MacLean HN. Two-dimensional echocardiographic assessment of the feline left atrium. J Vet Intern Med. 2006;20(1):111-9.

21. Todaro M, Choudhuri I, Belohlavek M, Jahangir A, Carerj S, Oreto L, et al. New echocardiographic techniques for evaluation of left atrial mechanics. Eur Heart J Cardiovasc Imaging. 2012;13(12):973-84.

22. Sugimoto $K$, Fujii $Y$, Sunahara $H$, Aoki T. Assessment of left ventricular longitudinal function in cats with subclinical hypertrophic cardiomyopathy using tissue Doppler imaging and speckle tracking echocardiography. J Vet Med Sci. 2015;77(9):1101-8

23. Suzuki R, Mochizuki Y, Yoshimatsu H, Teshima T, Matsumoto H, Koyama $H$. Determination of multidirectional myocardial deformations in cats with hypertrophic cardiomyopathy by using two-dimensional speckle-tracking echocardiography. J Feline Med Surg. 2017:19(12):1283-9.
24. Koffas H, Dukes-McEwan J, Corcoran B, Moran C, French A, Sboros V, et al. Colour M-mode tissue Doppler imaging in healthy cats and cats with hypertrophic cardiomyopathy. J Small Anim Pract. 2008;49(7):330-8.

25. Koffas H, Dukes-McEwan J, Corcoran B, Moran C, French A, Sboros V, et al, Pulsed tissue Doppler imaging in normal cats and cats with hypertrophic cardiomyopathy. J Vet Intern Med. 2006;20(1):65-77.

26. Rimbaş RC, Dulgheru RE, Vinereanu D. Methodological gaps in left atrial function assessment by $2 \mathrm{D}$ speckle tracking echocardiography. Arq Bras Cardiol. 2015;105(6):625-36.

27. Ahmed MK, Soliman MA, Reda AA, El-Ghani RSA. Assessment of left atrial deformation properties by speckle tracking in patients with systolic heart failure. Egypt Heart J. 2015;67(3):199-208.

28. Takano H, Isogai T, Aoki T, Wakao Y, Fujii Y. Feasibility of radial and circumferential strain analysis using 2D speckle tracking echocardiography in cats. J Vet Med Sci. 2015:77(2):193-201.

29. Visser LC, Sloan C, Stern JA. Echocardiographic assessment of right ventricular size and function in cats with hypertrophic cardiomyopathy. J Vet Intern Med. 2017;31(3):668-77.

30. Spalla I, Boswood A, Connolly DJ, Luis Fuentes V. Speckle tracking echocardiography in cats with preclinical hypertrophic cardiomyopathy. J Vet Intern Med. 2019;33(3):1232-41.

31. Nakamura K, Kawamoto S, Osuga T, Morita T, Sasaki N, Morishita K, et al. Left atrial strain at different stages of myxomatous mitral valve disease in dogs. J Vet Intern Med. 2017;31(2):316-25.

32. Roşca M, Lancellotti P, Popescu BA, Piérard LA. Left atrial function: pathophysiology, echocardiographic assessment, and clinical applications. Heart. 2011;97(23):1982-9.

33. Johns S, Nelson O, Gay J. Left atrial function in cats with left-sided cardiac disease and pleural effusion or pulmonary edema. J Vet Intern Med. 2012; 26(5):1134-9.

34. Vianna-Pinton R, Moreno CA, Baxter CM, Lee KS, Tsang TS, Appleton CP. Two-dimensional speckle-tracking echocardiography of the left atrium: feasibility and regional contraction and relaxation differences in normal subjects. J Am Soc Echocardiog. 2009;22(3):299-305.

35. Joos P, Porée J, Liebgott H, Vray D, Baudet M, Faurie J, et al. High-frame-rate speckle-tracking echocardiography. IEEE Trans Ultrason Ferroelectr Freq Control. 2018:65(5):720-8.

36. Nelson RW, Couto CG. Small Animal Internal Medicine. 5th ed. St.Louise: Elsevier; 2009. p. 145-58, 764.

37. Schober KE, Maerz I. Assessment of left atrial appendage flow velocity and its relation to spontaneous echocardiographic contrast in 89 cats with myocardial disease. J Vet Intern Med. 2006;20(1):120-30.

38. Disatian S, Bright J, Boon J. Association of age and heart rate with pulsedwave Doppler measurements in healthy, nonsedated cats. J Vet Intern Med. 2008;22(2):351-6.

39. Boon JA. Evaluation of Size, Function, and Hemodynamics. Veterinary Echocardiography. 2nd ed. Oxford: Wiley; 2011. p. 234 - 46,569 - 80.

40. Schober KE, Maerz I, Ludewig E, Stern JA. Diagnostic accuracy of electrocardiography and thoracic radiography in the assessment of left atrial size in cats: comparison with transthoracic 2-dimensional echocardiography. J Vet Intern Med. 2007;21(4):709-18.

41. Bland M. Clinical measurement. An introduction to medical statistics. 3rd ed. Oxford: Oxford medical publication; 2000. pp. 269-94.

\section{Publisher's Note}

Springer Nature remains neutral with regard to jurisdictional claims in published maps and institutional affiliations.

\section{Ready to submit your research? Choose BMC and benefit from:}

- fast, convenient online submission

- thorough peer review by experienced researchers in your field

- rapid publication on acceptance

- support for research data, including large and complex data types

- gold Open Access which fosters wider collaboration and increased citations

- maximum visibility for your research: over $100 \mathrm{M}$ website views per year

At BMC, research is always in progress.

Learn more biomedcentral.com/submission 